\title{
Mode-Locking, Quasi-Period and Chaos of Rotors Mounted on Nonlinear Bearings
}

\author{
YEON-PUN CHANG ${ }^{a}$, SHOOU-CHIAN JEN ${ }^{b}$, SHUN-HSU TU ${ }^{c}$, SHYH-SHYONG SHYR ${ }^{c}$ \\ and YUAN KANG ${ }^{\mathrm{c}, *}$ \\ ${ }^{a}$ Department of Mechanical Engineering, Chung Yuan Christian University, Chung Li, Taiwan 320, ROC; \\ bepartment of Mechanical Engineering, Sze Hai Institute of Technology and Commerce, ROC, \\ "Center for Aviation \& Space Technology, Industrial Technology Research Institute, ROC
}

(Received 24 April 1998; In final form 7 August 1998)

\begin{abstract}
This study is to investigate the dynamics of a rotor, with a single degree of freedom (SDOF), mounted on nonlinear bearings. This system has piecewise-linear stiffness and is subjected to a forcing excitation due to residual mass imbalance as well as a parametric one due to an axial periodic thrust. The frequencies for each individual parametric and forcing excitations are not equivalent, neither do they have a ratio of two simple integers. By using the fourthorder Runge-Kutta method a $J$-integral model, this strongly nonlinear system can be estimated for various parameters. The $J$-integral bifurcation can be analyzed by using the Poincaré maps, the frequency spectra, the response waveforms, and the Lyapunov exponents in order to illustrate the jump phenomenon, the frequency-locking, and the routes to chaos. Furthermore, the intra-systematic relationship can be determined by the frequencies of spontaneous sidebanding clusters.
\end{abstract}

Keywords: Nonlinear rotor dynamics, Bifurcation of $J$-integral, Frequency-locking motion, Quasi-periodic motion

\section{INTRODUCTION}

The nonlinear dynamics of the rotor-bearing systems is an important and practical problem because those systems frequently exist in many fields. The piecewise-linear systems having components with clearance have been introduced by the following ways. Firstly, the clearance factor is necessary at the stages of designing or assembling. Secondly, it can be caused by wear since the system has experienced intermittent motion for contacting and separating from other components. Last, the system might be connected through a backlash. These systems usually exhibit phenomena such as multiple solution regimes, superharmonic, subharmonic, quasiperiodic, and chaotic solutions.

The existance of nonlinear oscillations in the field of high-pressure and high-speed turbomachinary, motivates a lot of research studies. Experimental

* Corresponding author. Department of Mechanical Engineering, Chung Yuan Christian University, Chung Li, Taiwan 320, ROC. Tel.: 886(3) 4563171. Ext.: 4315. Fax: 886(3) 4563160. E-mail: Yuankang@ cycu.edu.tw. 
works have been conducted. For examples, observations including the subcritical superharmonic response, the supercritical subharmonic response, the spontaneous sidebanding, and the chaotic phenomenon have been shown in waveforms, frequency spectra, phase plane diagrams, and waterfall charts by Ehrich $(1988,1991,1992 a, b, 1995)$.

The conventional methods for analyzing piecewise-linear systems which have been subjected to the harmonic force can be analyzed as follows: (a) Padmanabhan and Singh (1995) have adapted an analytical method by using the parametric continuation scheme based on the shooting method. Although the occurrence of a period-doubling bifurcation which can be determined by the eigenvalues of the period-two has been resolved; however, the periodic solutions are hardly obtained. (b) Choi and Noah (1988, 1992), Kim and Noah (1991), Lau and Zhang (1992), and Narayanan and Sekar (1995) have used the harmonic balance method to determine the coefficients of solutions either directly or iteratively; however, these coefficients corresponding to the synchronous or multiple forcing frequency do not seem to converge easily to finite terms. Furthermore, the influence of initial conditions on steady-state solutions has been no longer unambiguous. Neither have these solutions been identical to the ones for the original equations of motion. Consequently the solutions were approximate ones only. (c) Mahfouz and Badrakhan $(1990 \mathrm{a}, \mathrm{b})$ have used the numerical integration method to solve two nonlinear systems which have merely the forcing excitations with harmonic frequencies. And they can determine the harmonic, the subharmonic, and the chaotic motions for various parameters.

This study is to investigate the dynamics of a SDOF rotor having piecewise-linear stiffness. Also, the system is subjected to a parametric excitation and a forcing excitation with a nonmultiple frequency ratio. This system is described by a secondorder differential equation and is solved by the fourth-order Runge-Kutta method in order to determine the stationary $J$-integral solutions for various parameters. The $J$-integral bifurcation can be analyzed by using the Poincare maps, the frequency spectra, the response waveforms, the phase portraits, and the Lyapunov exponents in order to illustrate the jump phenomenon, the frequencylocking, and the routes to chaos.

\section{EQUATIONS OF MOTION}

In a SDOF rotor as shown in Fig. 1, a typical one-mode oscillation is considered, and it can be governed by the following equation

$$
\begin{aligned}
& m \frac{\mathrm{d}^{2} y}{\mathrm{~d} t^{2}}+c \frac{\mathrm{d} y}{\mathrm{~d} t}+k_{1}\left(1-\mu_{2} \sin \left(\omega_{2} t\right)\right) y+F(y) \\
& \quad=p \sin \left(\omega_{1} t\right),
\end{aligned}
$$

in which piecewise-linear restoring force $F(y)$ is generated by a clearance $\varepsilon$, and is given by

$$
F(y)= \begin{cases}0 & \text { for }|y| \leq \varepsilon, \\ k_{2}(y-\varepsilon \operatorname{sgn} y) & \text { for }|y|>\varepsilon,\end{cases}
$$

where $m$ is the lump mass of a rotor, $c$ is the linear damping coefficient, $k_{1}$ and $k_{2}$ are the shaft stiffness and the restoring stiffness respectively, $\omega_{1}$ and $\omega_{2}$ are the forcing and the parametric frequencies, respectively, and $p$ and $k_{1} \mu_{2}$ are amplitudes of the forcing and the parametric excitations respectively. The parametric excitation is caused by an axial
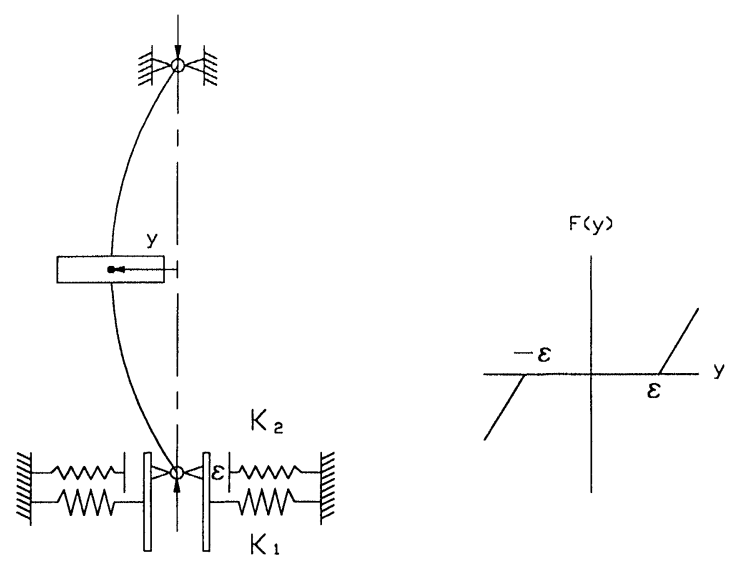

(a) a rotor system (b) restoring stiffness 
thrust, and the forcing excitation is caused by an unbalance force of the rotor.

By using the substitution of the following nondimensional variables,

$$
\begin{aligned}
x & =y / \varepsilon, \quad \tau=\omega_{0} t, \quad \omega_{0}=\sqrt{k_{1} / m}, \\
\mu_{1} & =p /\left(k_{1} \varepsilon\right), \quad \nu_{1}=\omega_{1} / \omega_{0}, \\
\nu_{2} & =\omega_{2} / \omega_{0}, \quad \gamma=c /\left(m \omega_{0}\right), \quad k=k_{2} / k_{1},
\end{aligned}
$$

Eqs. (1) and (2) gives

$$
\ddot{x}+\gamma \dot{x}+\left(1-\mu_{2} \sin \left(\nu_{2} \tau\right)\right) x+f(x)=\mu_{1} \sin \nu_{1} \tau,
$$

where

$$
f(x)= \begin{cases}0 & \text { for }|x| \leq 1, \\ k(x-\operatorname{sgn} x) & \text { for }|x|>1,\end{cases}
$$

and the superscript dot denotes the differentiation with respect to $\tau$.

Since the above equation has a strong nonlinear restoring force and the forcing frequency has a nonmultiple value via the parametric frequency, there has been no analytical method of solution. Thus, the fourth-order Runge-Kutta method is utilized. In the process of numerical integration, the value of $N$ is chosen from 200 to 600 for the time interval $\Delta \tau=2 \pi /\left(\nu_{1} N\right)$. The first forcing periods, which have long terms, were not recorded in order to avoid transient solutions. At the same time, a frequency spectrum is determined for the 400 periods state-steady solutions; also, the Poincaré map is obtained by sampling the stationary solutions over 2000 points $(x, \dot{x})$ for one forcing period $\left(T=2 \pi / \nu_{1}\right)$.

An effective integral of the response waveforms has been verified as a method for determining whether or not a transient solution can be lead to one of the steady solutions (Kang et al., 1998). This integral value monitored on the computer has been utilized in this study is

$$
J=\int_{0}^{2 \pi / \nu_{1}}[\dot{x}(\tau)]^{2} \mathrm{~d} \tau
$$

The initial conditions play an important role of determining the small orbit and large orbit; moreover, the multi-solutions for this kind system may coexist. In real systems, the actual disturbances cannot always be expressed in terms of the initial conditions. By using computer analysis with various initial conditions, the steady-state solutions of this system can be obtained and classified by $J$ integral. In general, the calculation of the $J$-integral for various initial conditions gives the number of values corresponding to the subharmonic number about the steady-state response. The large amplitudes should be distinguished from those small amplitudes which can be used to determine the occurrences of large orbits or small ones.

\section{MODE-LOCKED MOTION AND QUASI-PERIODIC MOTION}

The rotor system concerned in this study is characterized by two frequencies belonging to forcing and parametric excitations. The pattern of points on a Poincare map show the dependence on the numerical relationship between the two frequencies. If the ratio of the two frequencies can be expressed as the ratio of two integers (that is, as a "rational fraction,"), then the Poincaré section will consist of a finite number of points. This type of motion is often called frequency-locking, mode-locked, or phase-locking motion because one of the frequencies is locked over a finite control parameter range, and is a multiple integer of the other.

If the frequency ratio is irrational, the points on a Poincaré map will fill a continuous curve in the Poincaré plane. The motion can be described as quasi-periodic because the motion never repeats itself. However, the motion is not chaotic; rather, it is consisted of two or more periodic components whose presence can be determined by measuring the frequency spectrum of motion. In order to detect the difference between quasi-periodic and chaotic motions, the Lyapunov exponents are calculated by using the algorithm devised by Wolf et al. (1985). When the system has chaotic behavior, the 
calculated value of the most positive Lyapunov exponent is plotted as a function of the total average time which can be measured in units of the forcing period.

\section{NUMERICAL EXAMPLES}

For a rotor system of parameters at $\nu_{2}=3, k=7$, $\mu_{1}=0.25, \mu_{2}=0.17$, and $\gamma=0.07 ; J$-integral versus rotating speed $\nu_{1}$ is shown in Fig. 2. Small orbits occur in a range of $1.122<\nu_{1}<1.6$, large orbits occur in a range of $0.8<\nu_{1}<1.5$, and the coexisting motion occurs in a range of $1.1<\nu_{1}<1.5$. For the small orbit, one $J$-integral value indicates P1 motion. For the large orbit, a single $J$-integral value appeared in a range of $0.8<\nu_{1}<1.16$ indicates $\mathrm{P} 1$ motion; two $J$-integral values appeared in a range of $1.16<\nu_{1}<1.2$ indicate P2 motion; four $J$-integral values appeared in a range $1.2<\nu_{1}<1.208$ indicate P4 motion; and a range of speed over 1.21 appeared many $J$-integral values indicates chaotic motion. When the behavior is chaotic, the $J$-integral values seem to be smeared over the complete range of observed values. In particular, irregular $J$-integrals are scattered in the range of $1.1<\nu_{1}<1.122$ which then indicate tangle orbit.

The response waveform, the Poincaré maps, and the frequency spectra are shown in Fig. 3 for choosing frequency ratio $\nu_{2} / \nu_{1}$ as a value of $3 / \sqrt{2}$.

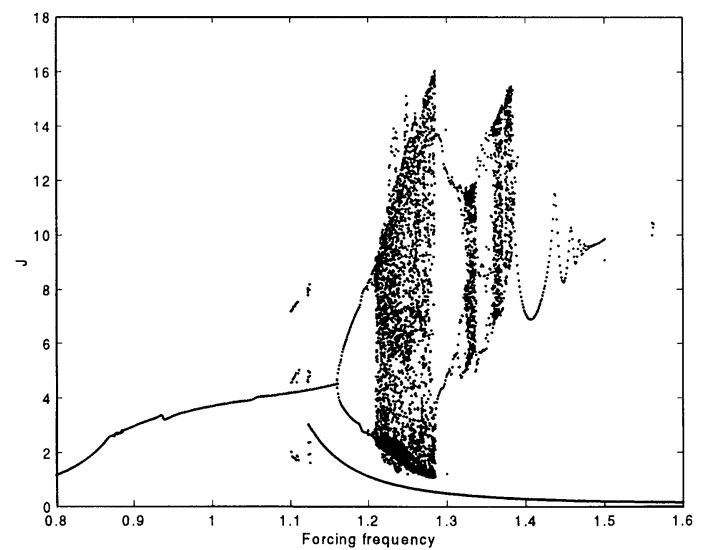

FIGURE 2 Bifurcation of $J$-integral versus rotating speed $\nu_{1} ; \nu_{2}=3, k=7, \mu_{1}=0.25, \mu_{2}=0.17, \gamma=0.07,0.8<\nu_{1}<1.6$.
Since the frequency ratio is irrational, the Poincaré map shows an almost-closed curve for a periodic response. Figure 4(a)-(p) show the frequency spectra and the Poincare maps for various rotating speeds in Fig. 2. The Poincaré maps of Fig. 4(a) and (b) are shown as closed curves corresponding to quasi-periodic motion, and eleven points correspond to period one motion for $\nu_{2} / \nu_{1}$ value being $3 / \sqrt{2}$ and 30/11, respectively. Figure 4(c), (d), (f), (h) show P1, P2, P4, and P16 motions corresponding to values of $1,2,4,16$ for the $J$-integral, respectively. Figure 4(i) and (j) show both small P1 motion and chaotic motions. In the rotating range with chaotic behavior, P2 and P4 motions are shown in Fig. 4(k) and (1). The tangle P3 orbit and large $\mathrm{P} 1$ orbit are coexisting at $\nu_{1}=1.101$ which can be shown in Fig. 4(m) and (n). Figure 4(o) and (p) show P3 motions on tangle orbit range.

The frequency spectra in Fig. 4 show that all peak frequencies can be described by

$$
f_{\mathrm{p}}=\left(n_{1} \nu_{2}+\nu_{1}\right) \pm n_{2} \Delta \omega_{n},
$$

where $\Delta \omega_{n}=\left|\nu_{2}-2 \nu_{1}\right| / n$, with $n$ integers, and $n_{1}, n_{2}=0,1,2, \ldots$

Thus, these motions are combinations of the $n_{1}$ th superharmonic components of $\nu_{2}$, the $\left(n_{2} / n\right)$ th subharmonic components of $\nu_{2}-2 \nu_{1}$ as well as the harmonic components of $\nu_{1}$. One may denote these motions by period $n$ or $\mathrm{P} n$ because of the corresponding number of the peak frequencies in each cluster.

The Feigenbaum universality constant has been derived for one-dimensional maps; however, many high-dimensional systems exhibit perioddoubling bifurcation which leads to chaos and can be observed of having a value of $(4.66920161 \ldots)$. As in this case, the Feigenbaum universality constant has been determined to be $(1.16-1.2)$ / $(1.2-1.208)=5.0$. Also, a chaotic behavior can be characterized by the positive value of the Lyapunov exponent. Figure 5 is shown that the largest Lyapunov exponent versus forcing period $T$ as the case of rotating speed at 1.21 and $\sqrt{2}$, respectively. 

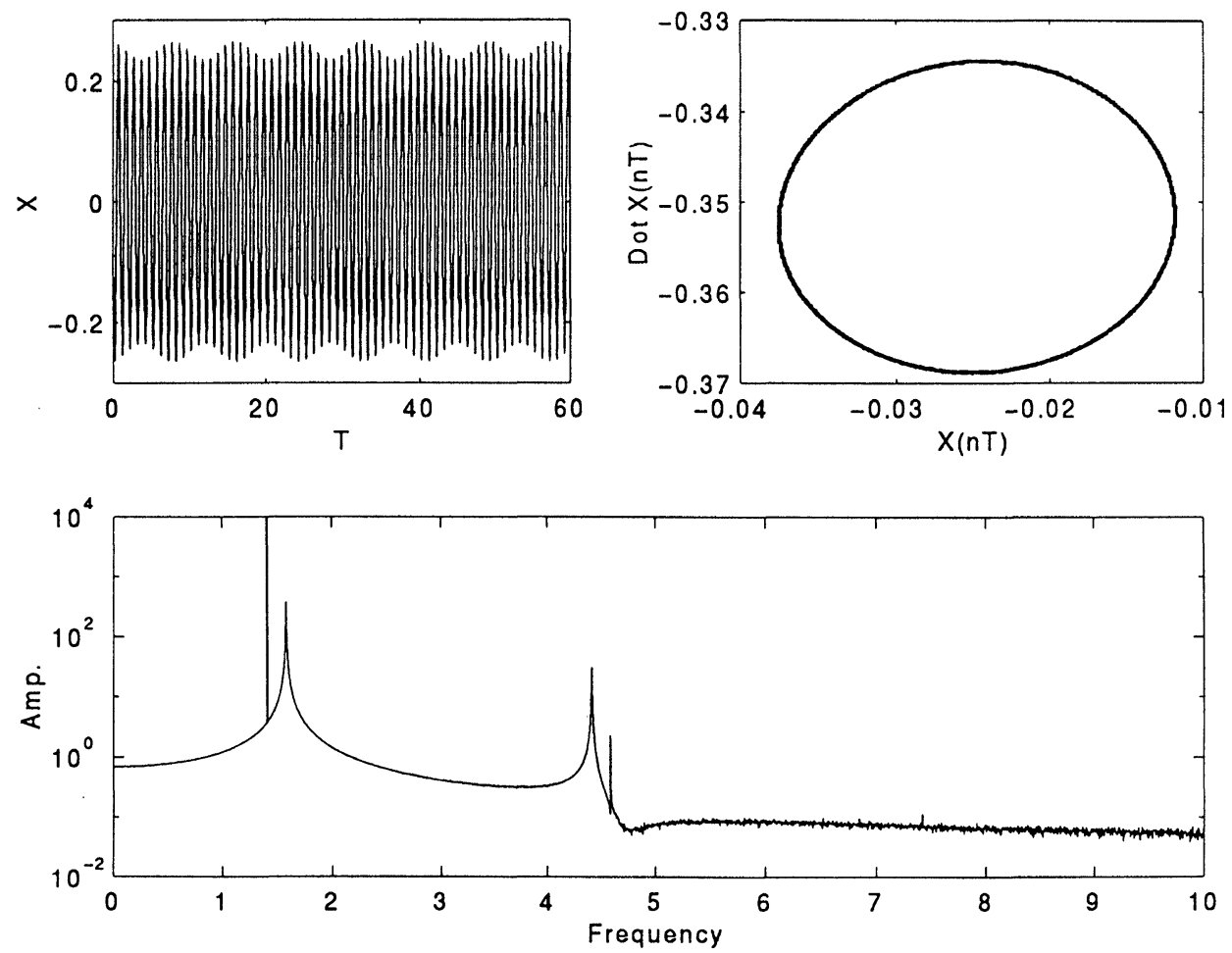

FIGURE 3 Response waveform (left). Poincaré map (right), and frequency spectrum (bottom) at $\nu_{1}=\sqrt{2}$.
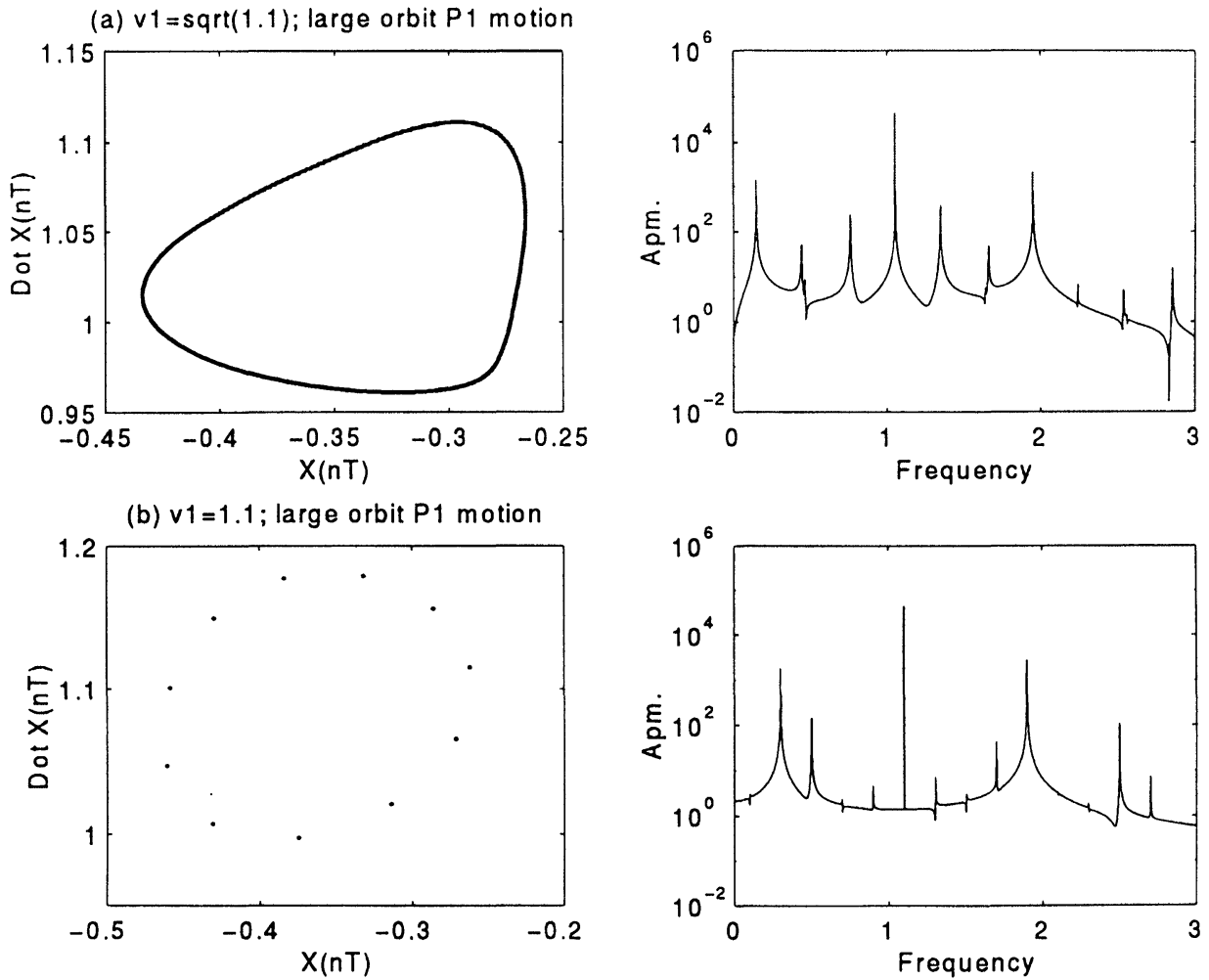

FIGURE 4(a) and (b) 
(c) $v 1=1.159 ;$ large orbit P1 motion

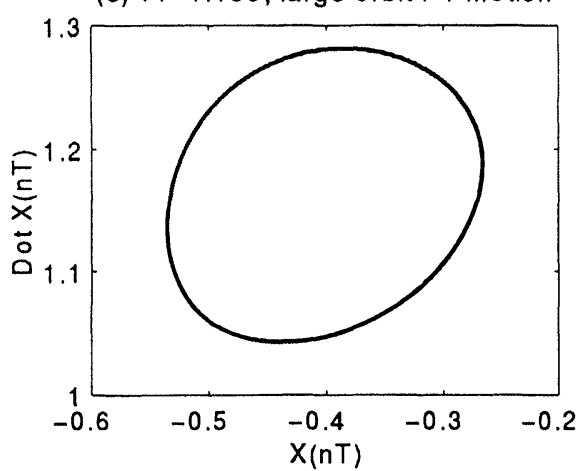

(d) v1=1.16; large orbit P2 motion

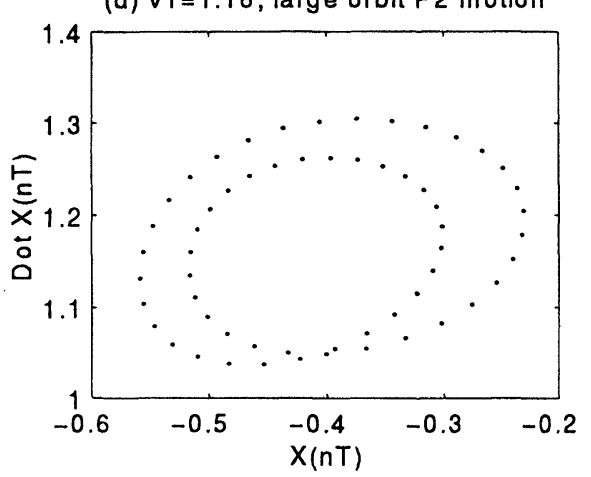

(e) $v 1=1.199 ;$ large orbit P2 motion
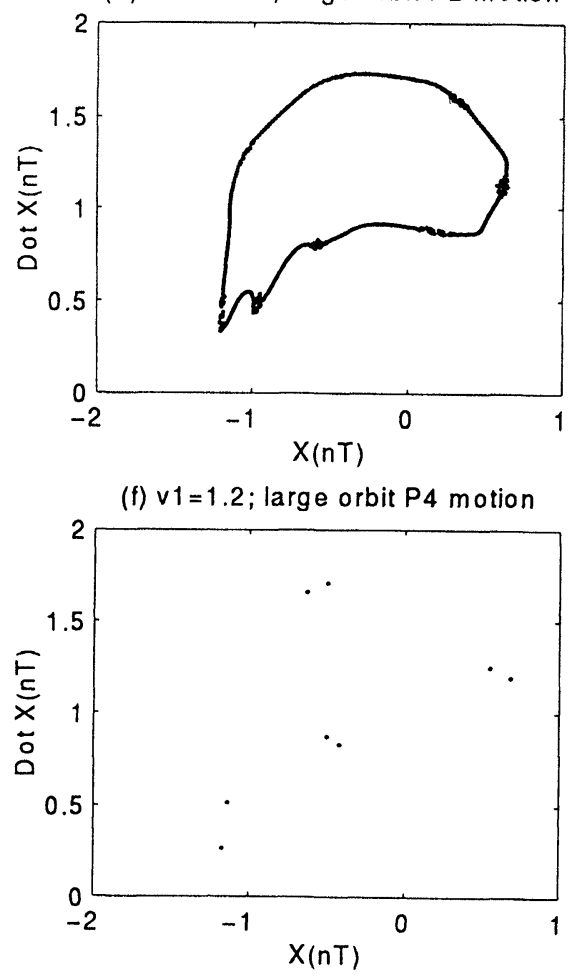
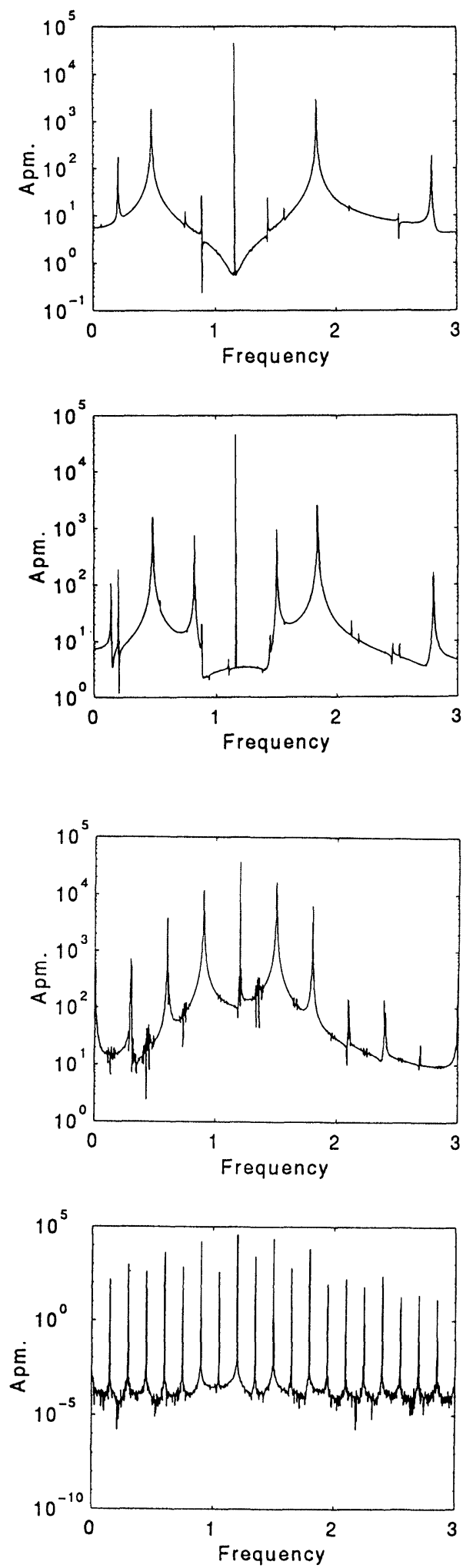

FIGURE 4(c)-(f) 

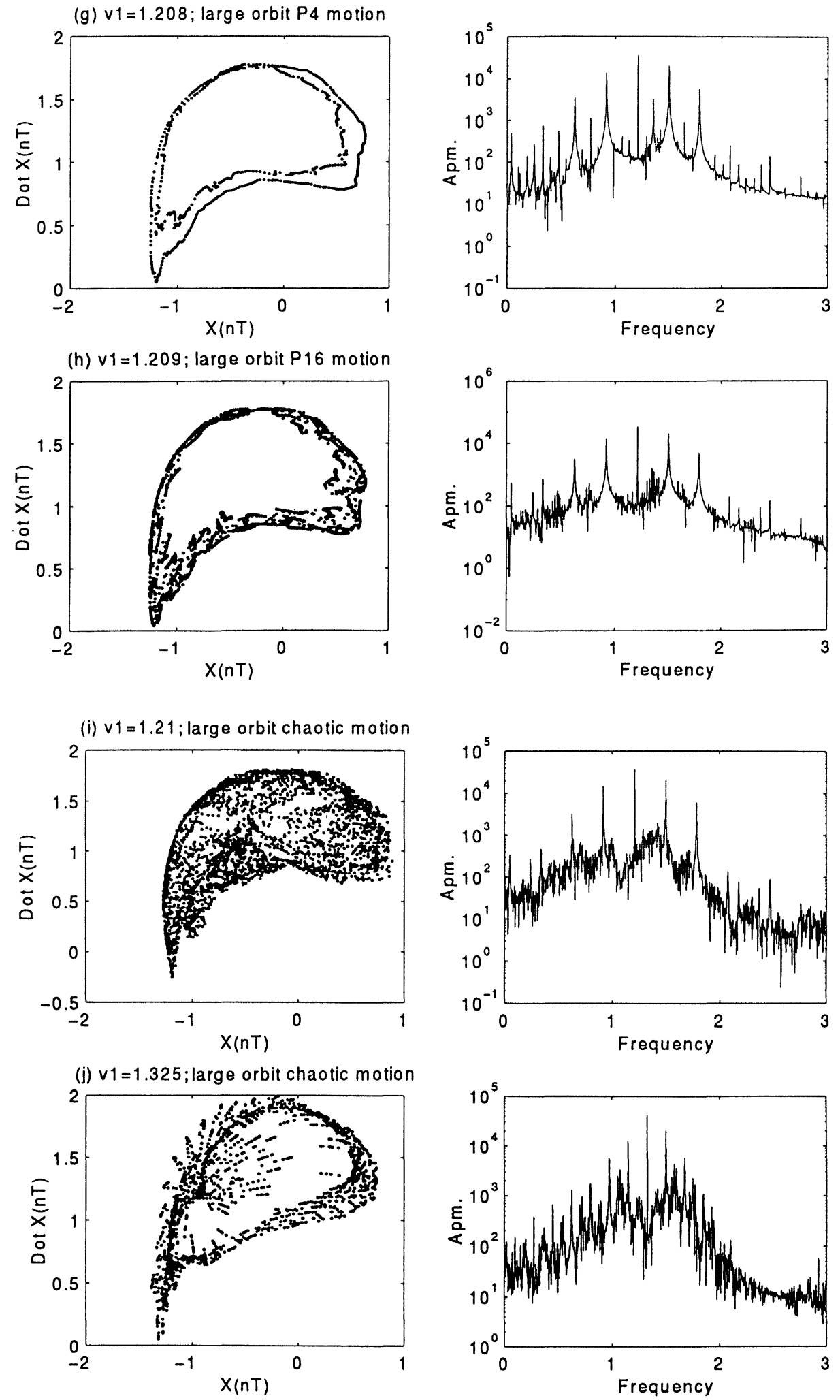

FIGURE 4(g)-(j) 

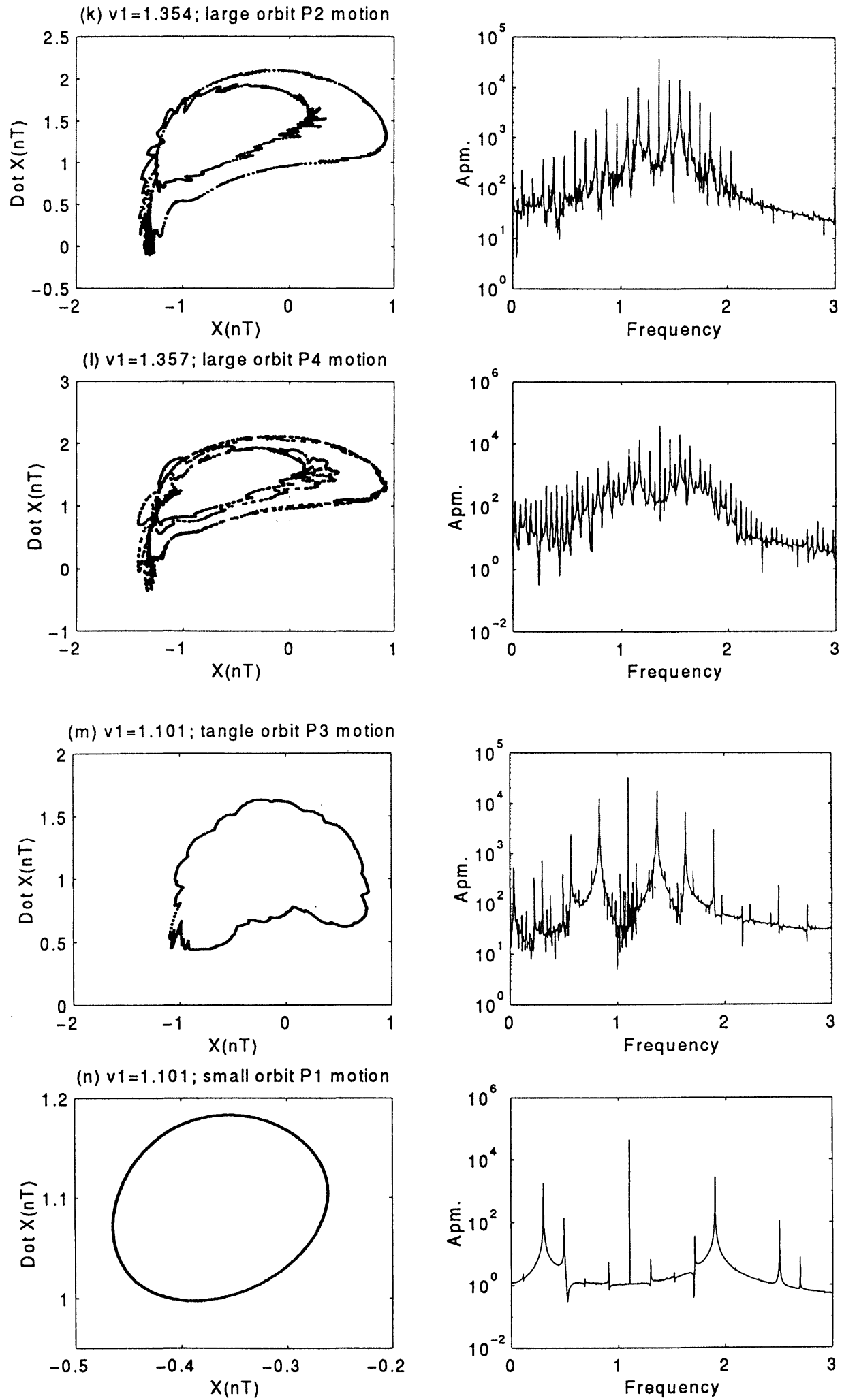

FIGURE 4(k)-(n) 
(o) V1=1.11; tangle orbit P3 motion

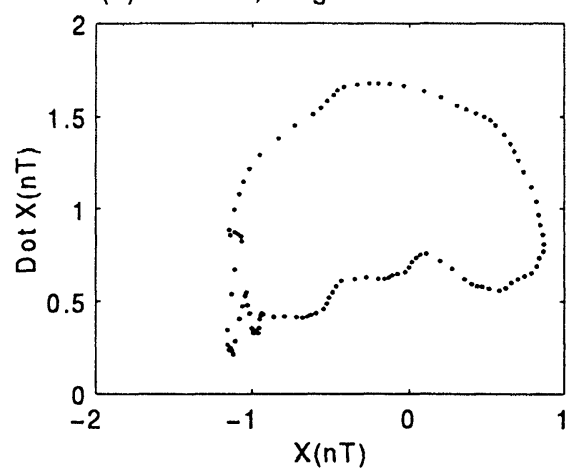

(p) $v 1=1.122 ;$ tangle orbit P3 motion

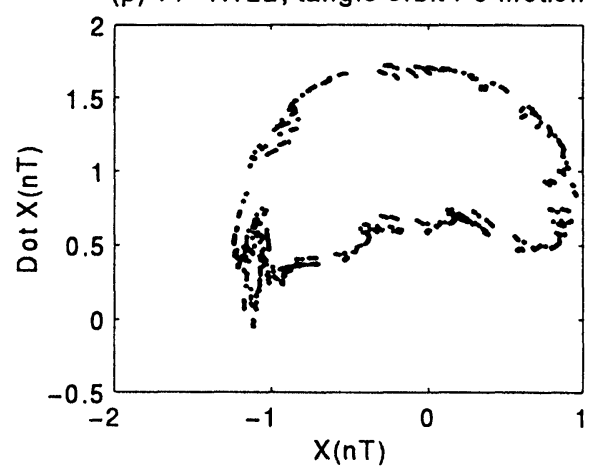

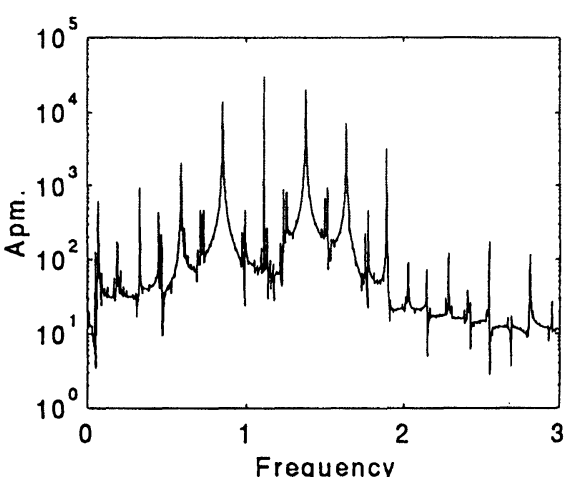

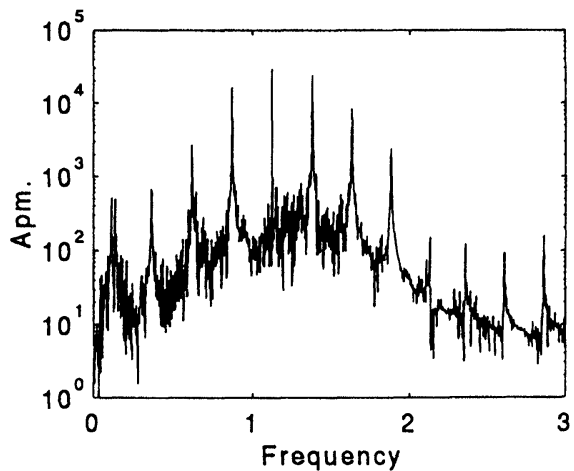

FIGURE 4(o) and (p)

FIGURE 4 Poincaré map (left), and frequency spectrum (right). (a) $\nu_{1}=\sqrt{1.1}$; large orbit P1 motion; (b) $\nu_{1}=1.1$; large orbit P1 motion; (c) $\nu_{1}=1.159$; large orbit P1 motion; (d) $\nu_{1}=1.116$; large orbit P2 motion; (e) $\nu_{1}=1.199$; large orbit P2 motion; (f) $\nu_{1}=1.2$; large orbit P4 motion; (g) $\nu_{1}=1.208$; large orbit P4 motion; (h) $\nu_{1}=1.209$; large orbit P16 motion; (i) $\nu_{1}=1.21$; large orbit chaotic motion; (j) $\nu_{1}=1.325$; large orbit chaotic motion; (k) $\nu_{1}=1.354$; large orbit P2 motion; (1) $\nu_{1}=1.357$; large orbit P4 motion; (m) $\nu_{1}=1.101$; tangle orbit P3 motion; (n) $\nu_{1}=1.101$; small orbit P1 motion; (o) $\nu_{1}=1.1$; tangle orbit P3 motion; (p) $\nu_{1}=1.122$; tangle orbit $\mathrm{P} 3$ motion.

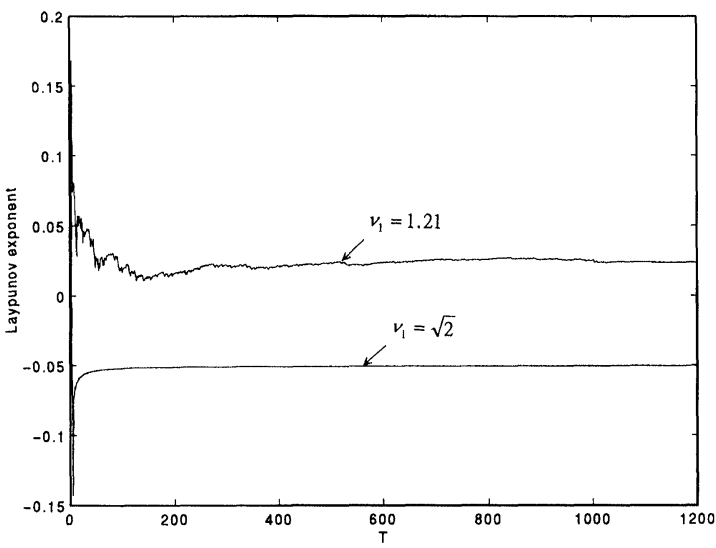

FIGURE 5 Lyapunov exponent versus $T ; \nu_{1}=1.21$, chaotic motion; $\nu_{1}=\sqrt{2}$, small orbit.

\section{CONCLUSION}

Oscillations of a strongly nonlinear rotor system which is subjected to both a forcing excitation due to an imbalance as well as a parametric excitation due to all axial periodic thrust has been investigated. The $J$-integral has been utilized to construct the bifurcations; thus, the small orbits and the large ones can be distinguished. Moreover, the jump phenomena, the subharmonics of various orders, the frequency-locking, the period $n$ motion, the perioddoubling cascade which leads to chaos can be distinguished. 


\section{NOMENCLATURE}

$c \quad$ damping coefficient $(\mathrm{N} \cdot \mathrm{s} / \mathrm{m})$

$f_{\mathrm{p}} \quad$ nondimensional frequency of the response peaks

$J \quad$ integral monitored; $J=\int_{0}^{2 \pi / \nu_{1}}[\dot{x}(\tau)]^{2} \mathrm{~d} \tau$ (dimensionless)

$k \quad$ nondimensional stiffness $k=k_{2} / k_{1}$

$k_{1} \quad$ shaft stiffness $(\mathrm{N} / \mathrm{m})$

$k_{2} \quad$ bearing stiffness $(\mathrm{N} / \mathrm{m})$

$m \quad$ lump mass of a rotor $(\mathrm{kg})$

$n, n_{1}, n_{2}$ integer

$p \quad$ amplitude of forcing excitation (N)

$P_{n} \quad$ period $n$ motion with $n$th order subharmonic frequencies

$t$ time (s)

$T \quad$ forcing period $T=2 \pi / \nu_{1}$ (dimensionless)

$x \quad$ nondimensional displacement; $x=y / \varepsilon$

$y \quad$ lateral displacement of SDOF rotor (m)

$\gamma \quad$ nondimensional damping coefficient; $\gamma=c /\left(m \omega_{0}\right)$

$\Delta \omega_{n} \quad \Delta \omega_{n}=\left|\nu_{2}-2 \nu_{1}\right| / n$ (dimensionless)

$\varepsilon \quad$ clearance $(\mathrm{m})$

$\mu_{1} \quad$ nondimensional amplitude of forcing excitation; $\mu_{1}=p /\left(k_{1} \varepsilon\right)$

$\mu_{2} \quad$ nondimensional amplitude of parametric excitation

$\nu_{1} \quad$ nondimensional forcing frequency; $\nu_{1}=\omega_{1} / \omega_{0}$

$\nu_{2} \quad$ nondimensional parametric frequency; $\nu_{2}=\omega_{2} / \omega_{0}$

$\tau \quad$ nondimensional time; $\tau=\omega_{0} t$

$\omega_{0} \quad$ fundamental natural frequency of the linearized rotor system; $\omega_{0}=\sqrt{k_{1} / m}$ $(\mathrm{rad} / \mathrm{s})$

$\omega_{1} \quad$ forcing frequency $(\mathrm{rad} / \mathrm{s})$

$\omega_{2} \quad$ parametric frequency $(\mathrm{rad} / \mathrm{s})$

\section{References}

Choi, Y.S. and Noah, S.T. (1988) Forced vibration of unsymmetric piecewise-linear systems, Journal of Sound and Vibration, 121, 117-128.

Choi, S.K. and Noah, S.T. (1992) Response and stability analysis of piecewise-linear oscillators under multi-forcing frequencies, Nonlinear Dynamics, 3, 105-121.

Ehrich, F.F. (1988) High Order Subharmonic Response of High Speed Rotors in Bearing Clearance, ASME Journal of Vibration, Acoustics, Stress, and Reliability in Design, 110, 9-16.

Ehrich, F.F. (1991) Some Observations of Chaotic Vibration Phenomena in High Speed Rotordynamics, ASME Journal of Vibration and Acoustics, 113, 50-57.

Ehrich, F.F. (1992a) Observations of Subcritical Superharmonic and Chaotic Response in Rotodynamics, ASME Journal of Vibration and Acoustics, 114, 93-100.

Ehrich, F.F. (1992b) Spontaneous Sidebanding in High Speed Rotor-dynamics, ASME Journal of Vibration and Acoustics, 114, 498-505.

Ehrich, F.F. (1995) Nonlinear Phenomena in Dynamic Response of Rotors in Anisotropic Mounting Systems, Transaction of the ASME Special 50th Anniversary Design Issue 117, 154-161.

Kang, Y., Jen, S.C. and Chang, Y.P. (1998) Strongly nonlinear oscillations of winding machines, part I: modal-locking motion and routes to chaos, Journal of Sound and Vibration, 209, 473-492.

Kim, Y.B. and Noah, S.T. (1991) Stability and bifurcation analysis of oscillators with piece-wise linear characteristics: a general approach, ASME Journal Applied Mechanics, 58, $545-553$.

Lau, S.L. and Zhang, W.S. (1992) Nonlinear vibrations of piecewise-linear systems by incremental harmonic balance method, ASME Journal of Applied Mechanics, 59, 153-160.

Mahfouz, I.A. and Badrakhan, F. (1990a) Chaotic behavior of some piecewise-linear systems part I: systems with set-up spring or with unsymmetric elasticity, Journal of Sound and Vibration, 143, 255-288.

Mahfouz, I.A. and Badrakhan, F. (1990b) Chaotic behavior of some piecewise-linear systems part II: systems with clearance, Journal of Sound and Vibration, 143, 289-328.

Narayanan, S. and Sekar, P. (1995) Periodic and chaotic responses of an SDF system with piecewise linear stiffness subjected to combined harmonic and flow induced excitations, Journal of Sound and Vibration, 184, 281-298.

Padmanabhan, C. and Singh, R. (1995) Analysis of periodically excited non-linear systems by a parametric continuation technique, Journal of Sound and Vibration, 184, 35-58.

Wolf, A., Swift, J.B., Swinney, H.L. and Vastano, J.A. (1985) Determining Lyapunov exponents from a time series, Physica D, 16, 285-317.

\section{Acknowledgments}

This study was supported by the National Science Council, the Republic of China, under grant number NBC 84-2212-E033-013. 


\section{ait \\ ENERGY MATERIALS}

M A N E Y publishing

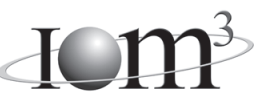

\section{Materials Science \& Engineering for Energy Systems}

Maney Publishing on behalf of the Institute of Materials, Minerals and Mining

The Institute of Materials, Minerals \& Mining

Economic and environmental factors are creating ever greater pressures for the efficient generation, transmission and use of energy. Materials developments are crucial to progress in all these areas: to innovation in design; to extending lifetime and maintenance intervals; and to successful operation in more demanding environments. Drawing together the broad community with interests in these areas, Energy Materials addresses materials needs in future energy generation, transmission, utilisation, conservation and storage. The journal covers thermal generation and gas turbines; renewable power (wind, wave, tidal, hydro, solar and geothermal); fuel cells (low and high temperature); materials issues relevant to biomass and biotechnology; nuclear power generation (fission and fusion); hydrogen generation and storage in the context of the 'hydrogen economy'; and the transmission and storage of the energy produced.

As well as publishing high-quality peer-reviewed research, Energy Materials promotes discussion of issues common to all sectors, through commissioned reviews and commentaries. The journal includes coverage of energy economics and policy, and broader social issues, since the political and legislative context influence research and investment decisions.

\section{CALL FOR PAPERS}

Contributions to the journal should be submitted online at http://ema.edmgr.com

To view the Notes for Contributors please visit: www.maney.co.uk/journals/notes/ema

Upon publication in 2006, this journal will be available via the Ingenta Connect journals service. To view free sample content online visit: www.ingentaconnect.com/content/maney

For further information please contact:

Maney Publishing UK

Tel: +44 (0)113 2497481 Fax: +44 (0)1132486983 Email: subscriptions@maney.co.uk

or

Maney Publishing North America

Tel (toll free): 8662975154 Fax: 6173546875 Email: maney@maneyusa.com

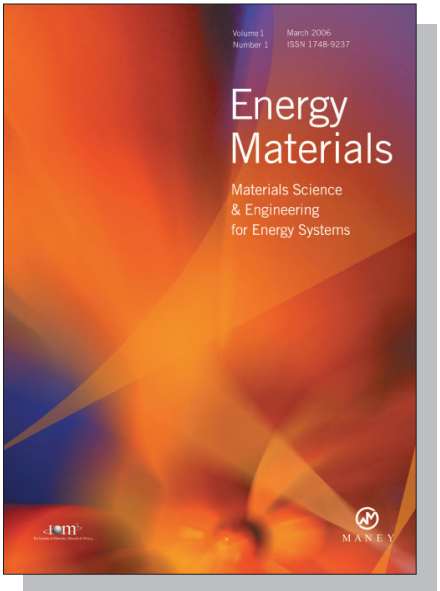

EDITORS

Dr Fujio Abe

NIMS, Japan

Dr John Hald, IPL-MPT, Technical University of Denmark, Denmark

Dr R Viswanathan, EPRI, USA

\section{SUBSCRIPTION INFORMATION}

Volume 1 (2006), 4 issues per year

Print ISSN: 1748-9237 Online ISSN: 1748-9245

Individual rate: $£ 76.00 / U S \$ 141.00$

Institutional rate: $£ 235.00 /$ US $\$ 435.00$

Online-only institutional rate: $£ 199.00 / U S \$ 367.00$

For special $\mathrm{IOM}^{3}$ member rates please email

subscriptions@maney.co.uk

\section{For further information or to subscribe online please visit www.maney.co.uk}



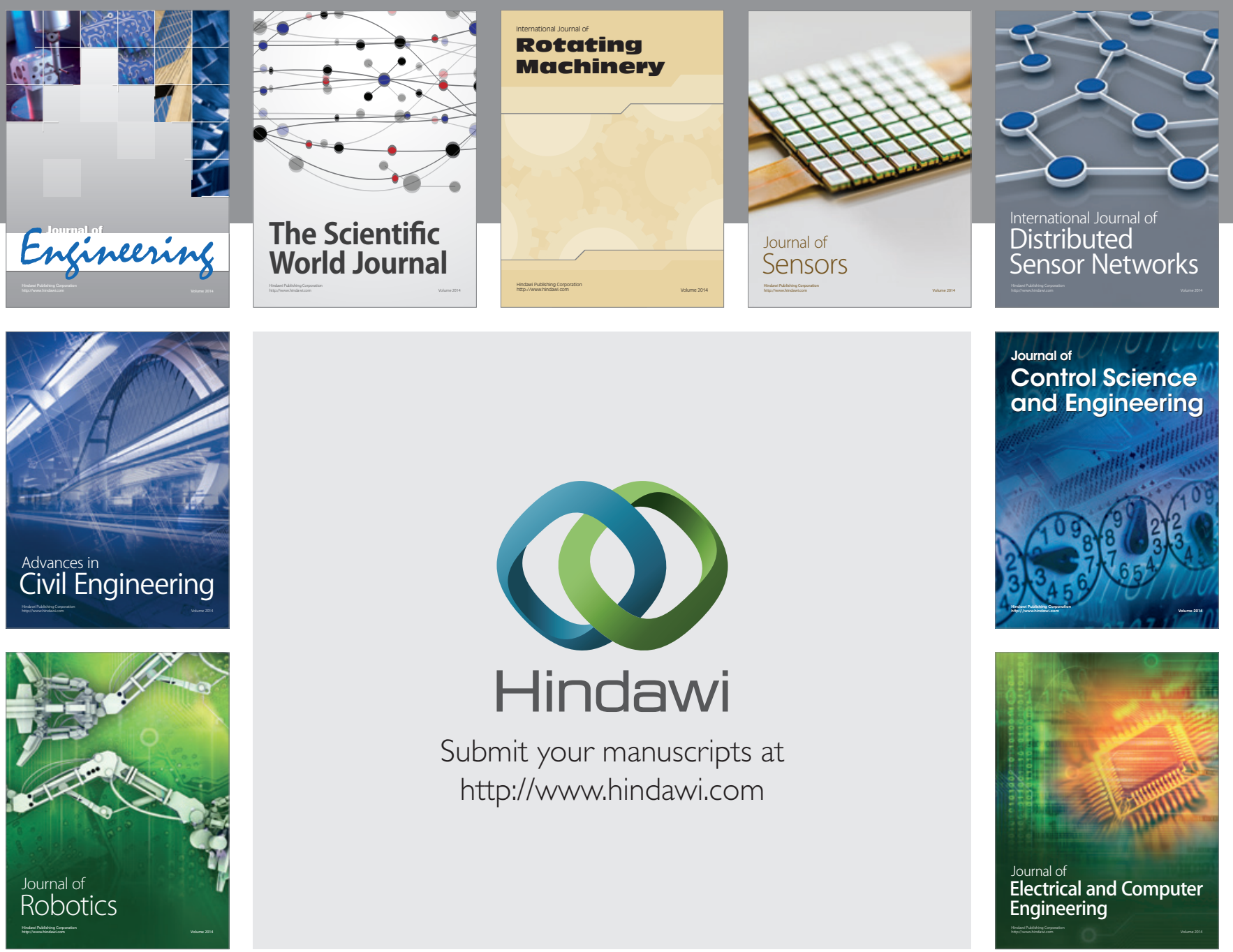

Submit your manuscripts at

http://www.hindawi.com
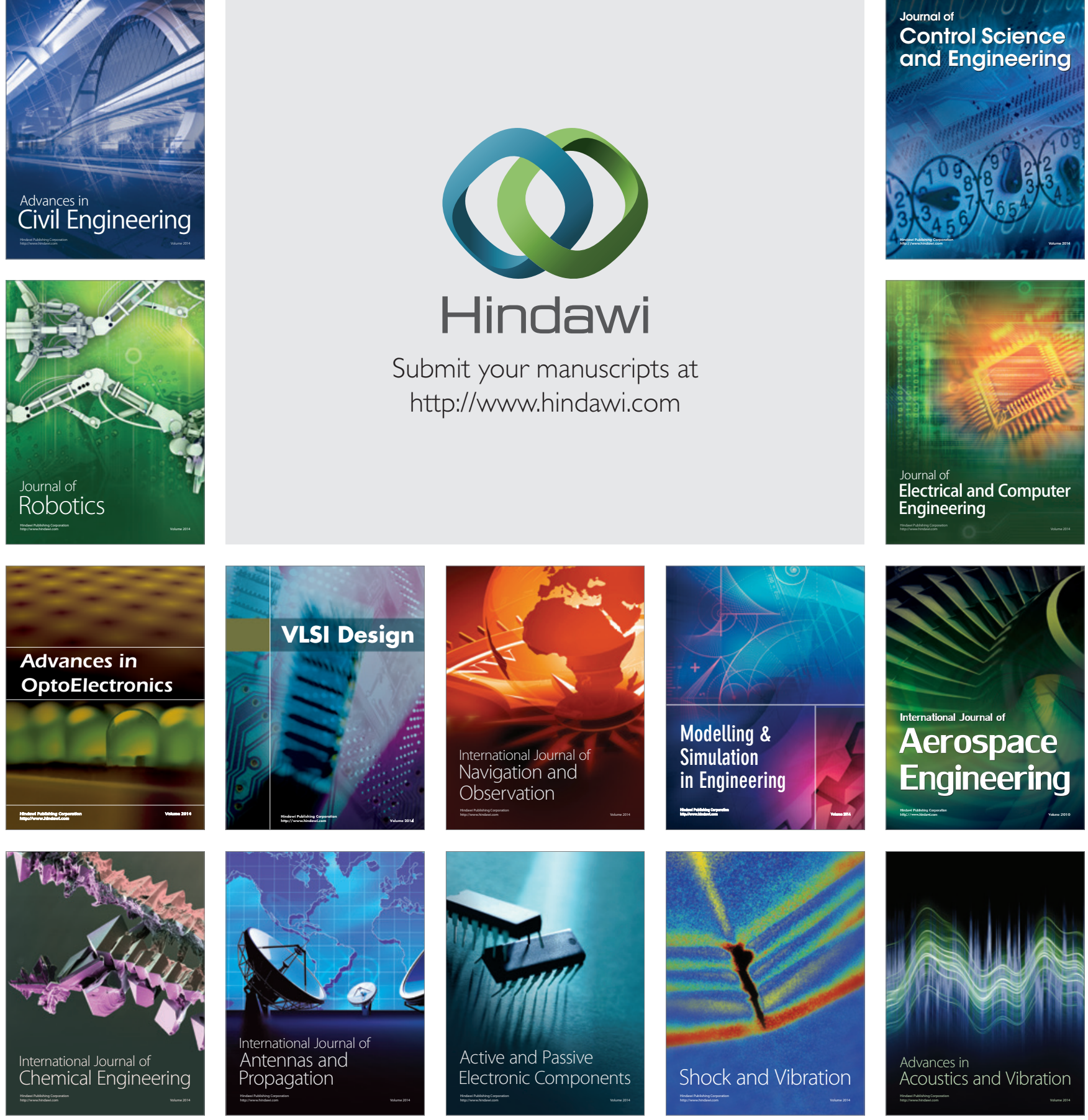